\title{
Erratum to: Levels of potential oral cancer salivary mRNA biomarkers in oral cancer patients in remission and oral lichen planus patients
}

\author{
Yi-Shing Lisa Cheng • Lee Jordan • Terry Rees • \\ Huey-Shys Chen • Lance Oxford • Ole Brinkmann • \\ David Wong
}

Published online: 31 October 2013

(C) Springer-Verlag Berlin Heidelberg 2013

Erratum to: Clin Oral Invest

DOI 10.1007/s00784-013-1041-0

The original version of this article inadvertently contained a mistake. The conflict of interest statement from co-author David Wong is missing.

Conflict of interest statement David Wong is co-founder of RNAmeTRIX Inc., a molecular diagnostic company. He holds equity in RNAmeTRIX, and serves as a company Director and Scientific Advisor. The University of California also holds equity in RNAmeTRIX. Intellectual property that David Wong invented and which was patented by the University of California has been licensed to RNAmeTRIX. Additionally, he is a paid consultant to PeriRx.

The online version of the original article can be found at http://dx.doi.org/ 10.1007/s00784-013-1041-0.

Y.-S. L. Cheng $(\bowtie) \cdot$ L. Jordan

Department of Diagnostic Sciences, Texas A\&M

University-Baylor College of Dentistry,

3302 Gaston Ave, Dallas, TX, USA

e-mail: ycheng@bcd.tamhsc.edu

T. Rees

Stomatology Center, Texas A\&M University-Baylor

College of Dentistry, Dallas, TX, USA

H.-S. Chen

College of Nursing, University of Toledo, Toledo, OH, USA

L. Oxford

Baylor University Medical Center, Dallas, TX, USA

O. Brinkmann

DENTSPLY DeTrey, Konstanz, Germany

D. Wong

School of Dentistry, University of California,

Los Angeles, CA, USA 